\title{
Variants of Interleukin-22 Gene Confer Predisposition to Autoimmune Thyroid Disease
}

\author{
Rong-hua Song, Qian Li, Wen Wang, Qiu-ming Yao, Xiao-qing Shao, and Jin-an Zhang \\ Department of Endocrinology, Jinshan Hospital of Fudan University, No. 1508 Longhang Road, Jinshan District, \\ Shanghai 201508, China \\ Correspondence should be addressed to Jin-an Zhang; zhanginan@hotmail.com
}

Received 16 March 2017; Accepted 13 June 2017; Published 3 August 2017

Academic Editor: Michael Horowitz

Copyright (c) 2017 Rong-hua Song et al. This is an open access article distributed under the Creative Commons Attribution License, which permits unrestricted use, distribution, and reproduction in any medium, provided the original work is properly cited.

\begin{abstract}
As there are no previous studies on the interleukin-22 (IL-22) variants in autoimmune thyroid disease (AITD), the present study aimed to explore the association between polymorphisms of IL-22 and the predisposition to AITD. The study had 975 AITD patients, including 639 Graves' disease (GD) and 336 Hashimoto's thyroiditis (HT) individuals and 851 healthy cohorts. Ligase detection reaction (LDR) and direct sequencing method were used for genotyping the IL-22 gene polymorphisms at rs2046068, rs2227478, rs2227485, rs11611206, and rs1179251. In comparison to female controls, genotype CC of rs1179251 was increased in the female AITD patients. Alleles C at rs2046068, C at rs2227478, and C at rs1179251 linked to the susceptibility of HT males. Genotype CC in rs1179251 was higher in male HT. Variants at rs2046068, rs2227478, and rs1179251 were associated with the AITD teenagers. Besides, genotype GG in rs11611206 was correlated with thyroid-associated ophthalmopathy (TAO). Moreover, allele G at rs11611206 was associated with decreased risk for TAO by $28.9 \%$. Similarly, genotype CC of rs 1179251 and genotype GG of rs11611206 were associated with Graves' ophthalmopathy (GO). Allele G in rs11611206 increased people with HT towards the predisposition of hypothyroidism. In conclusion, genetic variants of IL-22 are associated with the occurrence of AITD.
\end{abstract}

\section{Introduction}

Autoimmune thyroid disease (AITD), known to be the most common endocrine autoimmune disease, is an organic disease caused by immune irregulation related to the thyroid gland. AITD mainly includes two subtypes, Graves' disease (GD) and Hashimoto's thyroiditis (HT). The prevalence of AITD in the whole population has reached $2 \%-5 \%$ [1]. The presence of autoimmune antibodies, including antibody against TSH receptor (TRAb), anti-TPO antibody (TPOAb), and thyroglobulin antibody (TGAb), is the key immunological feature of AITD. AITD is also an immune polygenic disorder with a strong genetic component [2]. Recent advances in understanding the genetic basis for AITD documented the potential involvement of several genes in its pathophysiology. Polymorphisms in the genes, like TNFAIP3 [2, 3], IL-37 [4], and IRAK1/MECP2 [5], have been implicated in the occurrence and development of AITD.

Interleukin-22 (IL-22), belonging to the IL-10 cytokine family, is mainly expressed and secreted by Th22 (CD4+ IL22+) cells [6]. IL-22 gene polymorphisms have been found to be a risk factor for various autoimmune diseases, like psoriasis vulgaris [7] and celiac disease [8]. Recently, a study of ours revealed the protein level of interleukin-22 (IL-22) in serum, and the expression of IL-22 mRNA in peripheral blood mononuclear cells (PBMCs) was elevated in GD patients compared to healthy volunteers, implying IL-22 as an inflammatory cytokine participating in GD [9]. However, the relationship between IL-22 polymorphisms and AITD as yet remains unknown.

The present study was focused on investigating the allele frequencies, genotype distributions of the IL-22 variants, and 
TABLE 1: Clinical characteristics of AITD patients and controls.

\begin{tabular}{|c|c|c|c|c|}
\hline Clinical phenotype & AITD (\%) & GD (\%) & HT (\%) & Control (\%) \\
\hline Number & 975 & 639 & 336 & 851 \\
\hline \multicolumn{5}{|l|}{ Gender } \\
\hline Male & $233(23.90)$ & $190(29.73)$ & $43(12.80)$ & $284(33.37)$ \\
\hline Female & $742(76.10)$ & $449(70.27)$ & $293(87.20)$ & $567(66.63)$ \\
\hline Average age & $36.210 \pm 14.345$ & - & - & $38.700 \pm 8.945$ \\
\hline$\leq 18$ years & $105(10.77)$ & - & - & - \\
\hline With family history & $32(30.48)$ & - & - & - \\
\hline$\geq 19$ years & $870(89.23)$ & - & - & - \\
\hline With family history & $108(12.41)$ & - & - & - \\
\hline Age at diagnosis & $33.340 \pm 14.160$ & - & - & - \\
\hline Ophthalmopathy (+) & $118(12.10)$ & $112(17.53)$ & $6(1.79)$ & - \\
\hline Family history $(+)$ & $202(20.72)$ & - & - & - \\
\hline Smoking history (+) & $291(29.85)$ & - & - & - \\
\hline TRAb (+) & - & $639(100.00)$ & - & - \\
\hline TPOAb (+) & - & - & $267(79.53)$ & - \\
\hline TGAb $(+)$ & - & - & $176(52.30)$ & - \\
\hline Hypothyroidism & - & - & $171(50.89)$ & - \\
\hline
\end{tabular}

AITD: autoimmune thyroid disease; GD: Graves' disease; HT: Hashimoto's thyroiditis.

their correlation with the development of AITD and its clinical phenotypes.

\section{Material and Methods}

2.1. Ethics Statement. This study was approved by the institutional review board of Jinshan Hospital of Fudan University. The importance of the research was explained to all participants and written informed consent was obtained from each participant prior to the research.

2.2. Design and Setting. A case-control pilot was performed to define the allelic and genotypic profiles of polymorphisms (rs2046068, rs2227478, rs2227485, rs1179251, and rs11611206) within the IL-22 region in autoimmune thyroid disease in a cohort of Chinese origin and subphenotype groups.

2.3. Study Participants. In total, all the subjects (including established AITD patients and controls) were local ethnic Han Chinese. The study group enrolled 975 patients first diagnosed with AITD, including 639 Graves' disease and 336 Hashimoto's thyroiditis ones, and 851 healthy volunteers. The inclusion criterion to the AITD was consistent with that as detailed in our previous papers $[5,10]$. GD diagnosis was based on clinical manifestations, laboratory assessments (hyperthyroidism and positive TRAb, with or without positive TPOAb or TgAb), and diffuse goiter of the thyroid; HT was diagnosed based on the positive of either TPOAb or TgAb, with or without clinical and biochemical hypothyroidism and the presence of an enlarged thyroid. The suspected HT patients were further confirmed by the more accurate fine needle aspiration biopsies. The criterion for diagnosis of euthyroid in HT patients is normal TSH, FT3, and FT4. All AITD patients were collected from the Out-patient Clinic of Endocrinology of Jinshan Hospital of Fudan University. The sexand age-matched controls were from the Healthy Checkup Center of the same hospital, and they had no sibship with individuals in the patient group. The exclusion criteria for control subjects were positive thyroid antibodies against TPO tested by the immunochemiluminescence method (Roche Company, Shanghai, China) with high specificity and sensitivity. All the clinical and laboratory parameters were collected from patients' files and then analyzed.

2.4. Genotyping. Genomic DNA from AITD patients and healthy volunteers was extracted from $2 \mathrm{ml}$ of peripheral whole blood using commercial RelaxGene Blood DNA System provided by Tiangen Biotech Company (Beijing, China), in accordance with the manufacturer's protocol. All DNA samples measured the concentration and A260/A280 ratio by NanoDrop 2000 Spectrophotometer provided by Thermo Scientific Company (USA). Only the DNA samples with great purity and concentration were prepared for the next polymorphism genotyping.

In the present study, marker-tagging single-nucleotide polymorphisms (SNPs) which mapped the IL-22 gene were chosen from the previous studies $[7,8,11]$. At the same time, we chose marker-tagging SNPs from the Hapmap CHB data using the software named HaploView to meet the following criteria: minor allele frequency (MAF) $>0.05$, HardyWeinberg equilibrium (HWE) with $p>0.01$, and logarithm of odds (LOD) $>3.0$. For the IL-22 gene, we finally determined five loci covering the whole region of the gene to capture all the most common variants. In final, five tagSNPs, rs2046068, rs2227478, rs2227485, rs1179251, and rs11611206 of the IL-22 region, were selected. 
TABLE 2: Comparison of allele and genotype frequencies of IL-22 SNPs in AITD, GD, and HT patients and controls.

\begin{tabular}{|c|c|c|c|c|c|c|c|}
\hline \multirow{2}{*}{ SNP ID } & \multicolumn{4}{|c|}{ Groups } & \multicolumn{3}{|c|}{$P$ value $(\mathrm{OR} / 95 \% \mathrm{CI})$} \\
\hline & NC (\%) & AITD (\%) & GD (\%) & $\mathrm{HT}(\%)$ & AITD versus NC & GD versus NC & HT versus NC \\
\hline \multicolumn{8}{|c|}{ rs2046068 } \\
\hline AA & $559(65.69)$ & $622(63.79)$ & $419(65.57)$ & $203(60.42)$ & 0.495 & 0.67 & 0.211 \\
\hline $\mathrm{CC}$ & $31(3.64)$ & $45(4.62)$ & $29(4.54)$ & $16(4.76)$ & & & \\
\hline $\mathrm{AC}$ & $261(30.67)$ & $308(31.59)$ & $191(29.89)$ & $117(34.82)$ & & & \\
\hline $\mathrm{A}$ & $1379(81.02)$ & $1552(79.59)$ & $1029(80.52)$ & $523(77.83)$ & $\begin{array}{c}0.278 \\
(0.913 / 0.775-1.076)\end{array}$ & $\begin{array}{c}0.729 \\
(0.968 / 0.805-1.163)\end{array}$ & $\begin{array}{c}0.079 \\
(0.822 / 0.661-1.023)\end{array}$ \\
\hline $\mathrm{C}$ & $323(18.98)$ & $398(20.41)$ & $249(19.48)$ & $149(22.17)$ & & & \\
\hline \multicolumn{8}{|c|}{ rs2227478 } \\
\hline $\mathrm{TT}$ & $558(65.57)$ & $622(63.80)$ & $418(65.42)$ & $204(60.72)$ & 0.609 & 0.828 & 0.271 \\
\hline CC & $32(3.76)$ & $44(4.51)$ & $28(4.38)$ & $16(4.76)$ & & & \\
\hline TC & $261(30.67)$ & 309 (31.69) & $193(30.20)$ & $116(34.52)$ & & & \\
\hline $\mathrm{T}$ & $1377(80.90)$ & $1553(79.64)$ & $1029(80.52)$ & $524(77.98)$ & $\begin{array}{c}0.339 \\
(0.923 / 0.784-1.087)\end{array}$ & $\begin{array}{c}0.790 \\
(0.975 / 0.812-1.172)\end{array}$ & $\begin{array}{c}0.108 \\
(0.836 / 0.671-1.040)\end{array}$ \\
\hline $\mathrm{C}$ & $325(19.10)$ & $397(20.36)$ & $249(19.48)$ & $148(22.02)$ & & & \\
\hline \multicolumn{8}{|c|}{ rs2227485 } \\
\hline CC & $246(28.91)$ & $315(32.31)$ & $200(31.30)$ & $115(34.23)$ & 0.219 & 0.508 & 0.162 \\
\hline $\mathrm{TT}$ & $196(23.03)$ & $227(23.28)$ & $150(23.47)$ & $77(22.92)$ & & & \\
\hline $\mathrm{CT}$ & $409(48.06)$ & $433(44.41)$ & $289(45.23)$ & $144(42.85)$ & & & \\
\hline $\mathrm{C}$ & $901(52.94)$ & $1063(54.51)$ & $689(53.91)$ & $374(55.65)$ & $\begin{array}{c}0.341 \\
(1.065 / 0.935-1.214)\end{array}$ & $\begin{array}{c}0.598 \\
(1.040 / 0.899-1.203)\end{array}$ & $\begin{array}{c}0.232 \\
(1.116 / 0.932-1.335)\end{array}$ \\
\hline $\mathrm{T}$ & $801(47.06)$ & 887 (45.49) & 589 (46.09) & $298(44.35)$ & & & \\
\hline \multicolumn{8}{|c|}{ rs1179251 } \\
\hline $\mathrm{CC}$ & $354(41.60)$ & 427 (43.79) & $278(43.51)$ & $149(44.35)$ & 0.179 & 0.226 & 0.432 \\
\hline GG & 85 (9.99) & $115(11.80)$ & 77 (12.05) & $38(11.31)$ & & & \\
\hline CG & $412(48.41)$ & $433(44.41)$ & $284(44.44)$ & 149 (44.34) & & & \\
\hline $\mathrm{C}$ & $1120(65.80)$ & $1287(66.00)$ & $840(65.73)$ & $447(66.52)$ & $\begin{array}{c}0.901 \\
(1.009 / 0.879-1.157)\end{array}$ & $\begin{array}{c}0.965 \\
(0.997 / 0.855-1.161)\end{array}$ & $\begin{array}{c}0.741 \\
(1.032 / 0.855-1.247)\end{array}$ \\
\hline G & $582(34.20)$ & $663(34.00)$ & $438(34.27)$ & $225(33.48)$ & & & \\
\hline \multicolumn{8}{|c|}{ rs11611206 } \\
\hline GG & $686(80.61)$ & $782(80.21)$ & $506(79.19)$ & $276(82.14)$ & 0.298 & 0.342 & 0.273 \\
\hline $\mathrm{AA}$ & $4(0.47)$ & $11(1.12)$ & 7 (1.09) & $4(1.19)$ & & & \\
\hline GA & $161(18.92)$ & $182(18.67)$ & $126(19.72)$ & $56(16.67)$ & & & \\
\hline G & $1533(90.07)$ & $1746(89.54)$ & $1138(89.05)$ & $608(90.48)$ & $\begin{array}{c}0.596 \\
(0.944 / 0.761-1.170)\end{array}$ & $\begin{array}{c}0.364 \\
(0.896 / 0.707-1.135)\end{array}$ & $\begin{array}{c}0.765 \\
(1.047 / 0.774-1.417)\end{array}$ \\
\hline $\mathrm{A}$ & $169(9.93)$ & $204(10.46)$ & $140(10.95)$ & $64(9.52)$ & & & \\
\hline
\end{tabular}

SNP: single-nucleotide polymorphism; AITD: autoimmune thyroid disease; GD: Graves' disease; HT: Hashimoto's thyroiditis; NC: normal controls; OR: odds ratio; CI: confidence interval.

Genotyping was conducted using the ligase detection reaction (LDR) platform according to the manufacturer's instruction. Positive controls (duplication of the same DNA samples) and blank controls (blank samples without DNA) were both used in SNP genotyping to further ensure the reliable detection quality. All genotype results were recorded manually by different persons and inconsistent results were regenotyped by direct sequencing. Only SNPs that passed the $98 \%$ quality control threshold were subjected to the next statistical analyses. The SNPs with allele frequencies meeting Hardy-Weinberg equilibrium were included for next analysis. Specific primers of the five loci in the IL-22 gene are displayed as follows: (i) rs2046068: forward primer-AAAAGGCAACTCAG GTTGCG,

reverse primer-CTCCССТCAACAACTTAGAC;

(ii) rs2227478: forward primer-TGAGATGGCACAG ACCTAAG,

reverse primer-TCTGGCCACCTTCACAAATG;

(iii) rs2227485: forward primer-GTTTTGTCTTAGTA GAGTTC;

reverse primer- TGAGTCCGTGACCAAAATGC; 
TABLE 3: Allele and genotype distribution of IL-22 SNPs in different sex groups of AITD patients.

\begin{tabular}{|c|c|c|c|c|c|c|c|c|}
\hline \multirow{2}{*}{ Loci } & \multicolumn{4}{|c|}{ Male } & \multicolumn{4}{|c|}{ Female } \\
\hline & $\mathrm{NC}(\%)$ & AITD (\%) & $P$ value & OR/95\% CI & $\mathrm{NC}(\%)$ & AITD (\%) & $P$ value & OR/95\% CI \\
\hline \multicolumn{9}{|c|}{ rs2046068 } \\
\hline $\mathrm{AA}$ & $195(68.66)$ & $147(63.09)$ & 0.230 & & $364(64.20)$ & $475(64.02)$ & 0.955 & \\
\hline $\mathrm{CC}$ & $7(2.47)$ & $11(4.72)$ & & & $24(4.23)$ & $34(4.58)$ & & \\
\hline $\mathrm{AC}$ & $82(28.87)$ & $75(32.19)$ & & & $179(31.57)$ & $233(31.40)$ & & \\
\hline A & $472(83.10)$ & $369(79.18)$ & 0.108 & $0.774 / 0.566-1.058$ & 907 (79.98) & $1183(79.72)$ & 0.867 & $0.984 / 0.811-1.193$ \\
\hline C & $96(16.90)$ & $97(20.82)$ & & & $227(20.02)$ & $301(20.28)$ & & \\
\hline \multicolumn{9}{|c|}{ rs2227478 } \\
\hline $\mathrm{TT}$ & $195(68.66)$ & $148(63.52)$ & 0.329 & & $363(64.02)$ & $474(63.88)$ & 0.982 & \\
\hline $\mathrm{CC}$ & $8(2.82)$ & $11(4.72)$ & & & $24(4.23)$ & $33(4.45)$ & & \\
\hline TC & $81(28.52)$ & $74(31.76)$ & & & $180(31.75)$ & $235(31.67)$ & & \\
\hline $\mathrm{T}$ & $471(82.92)$ & $370(79.40)$ & 0.148 & $0.794 / 0.580-1.086$ & $906(79.89)$ & $1183(79.88)$ & 0.901 & $0.989 / 0.816-1.199$ \\
\hline $\mathrm{C}$ & $97(17.08)$ & $96(20.60)$ & & & $228(20.11)$ & $301(20.28)$ & & \\
\hline \multicolumn{9}{|c|}{ rs2227485 } \\
\hline CC & $76(26.76)$ & $64(27.47)$ & 0.880 & & $170(29.98)$ & $251(33.83)$ & 0.171 & \\
\hline $\mathrm{TT}$ & $70(24.65)$ & $53(22.74)$ & & & $126(22.22)$ & $174(23.45)$ & & \\
\hline $\mathrm{CT}$ & $138(48.59)$ & $116(49.79)$ & & & $271(47.80)$ & $317(42.72)$ & & \\
\hline $\mathrm{C}$ & $290(51.06)$ & $244(52.36)$ & 0.676 & $1.054 / 0.825-1.346$ & $611(53.88)$ & $819(55.19)$ & 0.505 & $1.054 / 0.903-1.231$ \\
\hline $\mathrm{T}$ & $278(48.94)$ & $222(47.64)$ & & & $523(46.12)$ & $665(44.81)$ & & \\
\hline \multicolumn{9}{|c|}{ rs 1179251} \\
\hline CC & $119(41.90)$ & $104(44.63)$ & 0.449 & & $235(41.45)$ & $323(43.53)$ & $0.048^{*}$ & \\
\hline GG & $31(10.92)$ & $18(7.73)$ & & & $54(9.52)$ & $97(13.07)$ & & \\
\hline CG & $134(47.18)$ & $111(47.64)$ & & & $278(49.03)$ & $322(43.40)$ & & \\
\hline $\mathrm{C}$ & $372(65.49)$ & $319(68.45)$ & 0.314 & $1.143 / 0.881-1.484$ & $748(65.96)$ & $968(65.23)$ & 0.696 & $0.968 / 0.823-1.139$ \\
\hline G & $196(34.51)$ & $147(31.55)$ & & & $386(34.04)$ & $516(34.77)$ & & \\
\hline \multicolumn{9}{|c|}{ rs11611206 } \\
\hline GG & $232(81.69)$ & $184(78.97)$ & 0.602 & & $454(80.07)$ & $598(80.59)$ & 0.387 & \\
\hline AA & $1(0.35)$ & $2(0.86)$ & & & $3(0.53)$ & $9(1.21)$ & & \\
\hline GA & $51(17.96)$ & $47(20.17)$ & & & $110(19.40)$ & $135(18.20)$ & & \\
\hline G & $515(90.67)$ & $415(89.06)$ & 0.391 & $0.837 / 0.558-1.256$ & $1018(89.77)$ & $1331(89.69)$ & 0.946 & $0.991 / 0.768-1.279$ \\
\hline A & $53(9.33)$ & $51(10.94)$ & & & $116(10.23)$ & $153(10.31)$ & & \\
\hline
\end{tabular}

${ }^{*} P<0.05$. SNP: single-nucleotide polymorphism; AITD: autoimmune thyroid disease; NC: normal controls; OR: odds ratio; CI: confidence interval.

(iv) rs11611206: forward primer-CACCTGTGAATCT CAGTTCC,

reverse primer-TCATGCTAGAGACCTGACAC;

(v) rs1179251: forward primer-GGTTGGGATCTTAG CTTGTC;

reverse primer-ACCTGCATTCTAGCCCTATC.

2.5. Clinical Subphenotype Analysis. Clinical subphenotype characteristic analyses were conducted by the case-only approach, in which basic allelic and genotypic examinations were performed by comparing minor allele and genotype frequency of cases with a specific subphenotype to the whole case group. The clinical subphenotypes include (1) onset age of the disease ( $\leq 18$ years old versus $\geq 19$ years old); (2) with or without thyroid eye disease, patients with mild or more serious than mild (moderate to severe) orbitopathy were diagnosed as ophthalmopathy according to EUGOGO criteria [12]; (3) with or without hypothyroidism in the HT group.

2.6. Statistical Analysis. All of the data were mainly tested with the SPSS Statistics 20.0 software (IBM, Armonk, NY). Linkage disequilibrium (LD) test and haplotype frequency calculation were carried on using the software of HaploView 4.2. Evaluation of Hardy-Weinberg equilibrium (HWE) of the five loci using the chi-square $\left(\chi^{2}\right)$ test was done prior to performing an association statistics. And exploration of the association was performed by comparing allele and genotype between AITD subjects and healthy ones using the $\chi^{2}$ test or Fisher's exact analysis. Linkage disequilibrium (LD) analysis was performed for the five loci using the HaploView program. Two-tailed $P$ values $<0.05$ were considered of statistical significance in all assessments. Odds ratio (OR) was to assess the correlation between each locus and AITD. 


\section{Results}

3.1. Characteristics of Chinese AITD and Control Subjects. Table 1 showed all the phenotype information about our cohort. We studied 975 patients with AITD (639 GD cases and 336 HT cases) and 851 healthy volunteers of Chinese origin. In the AITD group, there were 742 female and 233 male patients, and the average age of the patients was $36.210 \pm 14.345$ years old. The median age at diagnosis was $33.340 \pm 14.160$ years old (range 1-77 years). There were 202 subjects with family history in the AITD group with a percentage of $20.72 \%$. Additionally, there were 105 teenagers in this case group with a percentage of $10.77 \%$. The percentages of family history were $30.48 \%$ in those with onset age $\leq 18$ years old and $12.41 \%$ in those subjects with onset age $\geq 19$ years old. Besides, we unraveled that there were 118 AITD patients with ophthalmopathy (with a percentage of $12.10 \%$ ), including $112 \mathrm{GD}$ patients. TRAb were positive in all GD patients. In addition, in the HT subgroup, 171 subjects had hypothyroidism (at a rate of 50.89\%), 79.53\% cases were with positive TPOAb (267 ones), and $52.30 \%$ cases were with positive TGAb (176 ones). In the control group, there were 567 female and 284 male patients and the average age was $38.700 \pm 8.945$ years old. No statistical significance was found in comparing the age and gender in the AITD, GD, and HT groups to the control group $(P>0.05$, data not shown).

3.2. Hardy-Weinberg Equilibrium Analysis. IL-22 SNP genotyping identified the genotypes $\mathrm{A} / \mathrm{A}, \mathrm{A} / \mathrm{C}$, and $\mathrm{C} / \mathrm{C}$ at rs2046068; T/T, T/C, and $\mathrm{C} / \mathrm{C}$ at $\mathrm{rs} 2227478 ; \mathrm{C} / \mathrm{C}, \mathrm{C} / \mathrm{T}$, and $\mathrm{T} / \mathrm{T}$ at rs2227485; C/C, C/G, and G/G at rs1179251; and G/G, G/A, and A/A at rs11611206. According to the genotyping results, the distributions of all genotypes at rs2046068, rs2227478, rs2227485, rs1179251, and rs11611206 in the IL-22 gene in the control group met the expectations of Hardy-Weinberg equilibrium $(P>0.01)$. Besides, we detected linkage disequilibrium (LD) between any two loci in the five ones of IL-22. The $D$ values between any two loci among the four SNPs (rs2046068, rs2227478, rs2227485, and rs1179251) were obtained larger than 0.9 , which shows that the four loci were in strong linkage disequilibrium.

3.3. Allelic and Genotypic Analysis. There was no significant association between the IL-22 gene polymorphisms (rs2046068, rs2227478, rs2227485, rs1179251, and rs11611206) and AITD, GD, and HT ( $P$ values all larger than $0.05)$, which were shown in Table 2. But the frequency of allele A of rs2046068 (77.83\%) in HT patients was lower than that in controls (81.02\%), although this association failed to reach significant probabilities $(P=0.079)$. Moreover, as seen in Table 3, locus analysis of the alleles and genotype distribution in female AITD patients and female controls indicated that genotype CC of rs1179251 linked to the susceptibility of AITD $(P=0.048)$. But we found no evidence for an association between the allele and genotype distribution and the risk of AITD in male patients $(P>0.05$, shown in Table 3).
TABLE 4: Allele and genotype analysis of IL-22 SNPs in male patients with HT.

\begin{tabular}{|c|c|c|c|c|}
\hline \multirow{2}{*}{ Loci } & \multicolumn{4}{|c|}{ Male } \\
\hline & NC (\%) & HT (\%) & $P$ value & OR/95\% CI \\
\hline \multicolumn{5}{|c|}{ rs2046068 } \\
\hline AA & $195(68.66)$ & $22(51.16)$ & 0.084 & \\
\hline $\mathrm{CC}$ & $7(2.47)$ & $2(4.65)$ & & \\
\hline $\mathrm{AC}$ & $82(28.87)$ & 19 (44.19) & & \\
\hline A & $472(83.10)$ & $63(73.26)$ & $0.027^{*}$ & $\begin{array}{c}0.557 \\
(0.329-0.942)\end{array}$ \\
\hline $\mathrm{C}$ & $96(16.90)$ & $23(26.74)$ & & \\
\hline \multicolumn{5}{|c|}{ rs2227478 } \\
\hline $\mathrm{TT}$ & $195(68.66)$ & $22(51.16)$ & 0.086 & \\
\hline $\mathrm{CC}$ & $8(2.82)$ & $2(4.65)$ & & \\
\hline TC & $81(28.52)$ & $19(44.19)$ & & \\
\hline $\mathrm{T}$ & $471(82.92)$ & $63(73.26)$ & $0.031^{*}$ & $\begin{array}{c}0.564 \\
(0.334-0.954)\end{array}$ \\
\hline $\mathrm{C}$ & 97 (17.08) & $23(26.74)$ & & \\
\hline \multicolumn{5}{|c|}{ rs2227485 } \\
\hline $\mathrm{CC}$ & $76(26.76)$ & $11(25.58)$ & 0.984 & \\
\hline $\mathrm{TT}$ & $70(24.65)$ & $11(25.58)$ & & \\
\hline CT & $138(48.59)$ & $21(48.84)$ & & \\
\hline $\mathrm{C}$ & $290(51.06)$ & $43(50.00)$ & 0.855 & $\begin{array}{c}0.959 \\
(0.609-0.509)\end{array}$ \\
\hline $\mathrm{T}$ & $278(48.94)$ & $43(50.00)$ & & \\
\hline \multicolumn{5}{|c|}{ rs1179251 } \\
\hline $\mathrm{CC}$ & $119(41.90)$ & $23(53.49)$ & $0.007^{*}$ & \\
\hline GG & $31(10.92)$ & 0 & & \\
\hline CG & $134(47.18)$ & $20(46.51)$ & & \\
\hline $\mathrm{C}$ & 372 (65.49) & $66(76.74)$ & $0.039^{*}$ & $\begin{array}{c}1.739 \\
(1.024-2.952)\end{array}$ \\
\hline G & $196(34.51)$ & $20(23.26)$ & & \\
\hline \multicolumn{5}{|c|}{ rs11611206 } \\
\hline GG & 232 (81.69) & $33(76.74)$ & 0.396 & \\
\hline AA & $1(0.35)$ & $1(2.33)$ & & \\
\hline GA & $51(17.96)$ & $9(20.93)$ & & \\
\hline G & $515(90.67)$ & $75(87.21)$ & 0.314 & $\begin{array}{c}0.702 \\
(0.351-1.403)\end{array}$ \\
\hline A & $53(9.33)$ & $11(12.79)$ & & \\
\hline
\end{tabular}

${ }^{*} P<0.05$. SNP: single-nucleotide polymorphism; HT: Hashimoto's thyroiditis; NC: normal controls; OR: odds ratio; CI: confidence interval.

Similarly, there were no differences in the allele and genotype distributions in male GD and female GD versus those in corresponding controls ( $P$ values all larger than 0.05 , data not shown). Nevertheless, as displayed in Table 4, compared to that in male controls, allele C at rs2046068, allele C at 2227478, and allele $C$ at 1179251 can increase the risk towards the predisposition of HT in males $(P=0.0 .27$, $P=0.031$, and $P=0.039$, resp.). The difference in allele frequency observed for the polymorphism in rs1179251 was further verified by genotype probabilities, and genotype CC of rs1179251 was also associated with HT in male subjects $(P=0.007)$. 
TABLE 5: Allele and genotype frequencies of IL-22 SNPs with onset age and ophthalmopathy in AITD patients.

\begin{tabular}{|c|c|c|c|c|c|c|}
\hline \multirow{2}{*}{ SNP } & \multicolumn{3}{|c|}{ Onset age of AITD patients } & \multicolumn{3}{|c|}{ Ophthalmopathy } \\
\hline & $\geq 19(\%)$ & $\leq 18(\%)$ & $P$ value $(\mathrm{OR} / 95 \% \mathrm{CI})$ & Without (\%) & With (\%) & $P$ value $(\mathrm{OR} / 95 \% \mathrm{CI})$ \\
\hline \multicolumn{7}{|c|}{ rs2046068 } \\
\hline AA & $556(63.91)$ & $66(62.86)$ & $0.034^{*}$ & $547(63.83)$ & 75 (63.56) & 0.758 \\
\hline $\mathrm{CC}$ & $35(4.02)$ & $10(9.52)$ & & $38(4.43)$ & $7(5.93)$ & \\
\hline $\mathrm{AC}$ & $279(32.07)$ & $29(27.62)$ & & $272(31.74)$ & $36(30.51)$ & \\
\hline A & $1391(79.94)$ & $161(76.67)$ & $0.266(0.824 / 0.586-1.159)$ & $1366(79.70)$ & $186(78.81)$ & $0.752(0.948 / 0.679-1.323)$ \\
\hline $\mathrm{C}$ & $349(20.06)$ & $49(23.33)$ & & $348(20.30)$ & $50(21.19)$ & \\
\hline \multicolumn{7}{|c|}{ rs2227478 } \\
\hline $\mathrm{TT}$ & $556(63.91)$ & $66(62.86)$ & $0.028^{*}$ & $547(63.83)$ & $75(63.56)$ & 0.719 \\
\hline $\mathrm{CC}$ & $34(3.91)$ & $10(9.52)$ & & $37(4.32)$ & $7(5.93)$ & \\
\hline TC & $280(32.18)$ & $29(27.62)$ & & $273(31.85)$ & $36(30.51)$ & \\
\hline $\mathrm{T}$ & $1392(80.00)$ & $161(76.67)$ & $0.257(0.821 / 0.584-1.155)$ & $1367(79.75)$ & $186(78.81)$ & $0.736(0.944 / 0.676-1.318)$ \\
\hline C & $348(20.00)$ & $49(23.33)$ & & $347(20.25)$ & $50(21.19)$ & \\
\hline \multicolumn{7}{|c|}{ rs2227485 } \\
\hline $\mathrm{CC}$ & $287(32.99)$ & $28(26.67)$ & 0.421 & $279(32.56)$ & $36(30.51)$ & 0.904 \\
\hline $\mathrm{TT}$ & $201(23.10)$ & $26(24.76)$ & & $199(23.22)$ & $28(23.73)$ & \\
\hline $\mathrm{CT}$ & $382(43.91)$ & $51(48.57)$ & & $379(44.22)$ & $54(45.76)$ & \\
\hline $\mathrm{C}$ & $956(54.94)$ & $107(50.95)$ & $0.273(0.852 / 0.640-1.135)$ & $937(54.67)$ & $126(53.39)$ & $0.712(0.950 / 0.723-1.248)$ \\
\hline $\mathrm{T}$ & $784(45.06)$ & $103(49.05)$ & & $777(45.33)$ & $110(46.61)$ & \\
\hline \multicolumn{7}{|c|}{ rs1179251 } \\
\hline CC & $372(42.76)$ & $55(52.38)$ & 0.120 & $368(42.94)$ & $59(50.00)$ & 0.116 \\
\hline GG & $107(12.30)$ & $8(7.62)$ & & $98(11.44)$ & $17(14.41)$ & \\
\hline CG & $391(44.94)$ & $42(40.00)$ & & $391(45.62)$ & $42(35.59)$ & \\
\hline $\mathrm{C}$ & $1135(65.23)$ & $152(72.38)$ & $0.039^{*}(1.397 / 1.016-1.920)$ & $1127(65.75)$ & $160(67.80)$ & $0.534(1.097 / 0.820-1.466)$ \\
\hline G & $605(34.77)$ & $58(27.62)$ & & $587(34.25)$ & $76(32.20)$ & \\
\hline \multicolumn{7}{|c|}{ rs11611206 } \\
\hline GG & $694(79.77)$ & $88(83.81)$ & 0.386 & $696(81.21)$ & $86(72.88)$ & $0.023^{*}$ \\
\hline AA & $11(1.26)$ & $0(0)$ & & $11(1.28)$ & $0(0)$ & \\
\hline GA & $165(18.97)$ & $17(16.19)$ & & $150(17.51)$ & $32(27.12)$ & \\
\hline G & $1553(89.25)$ & $193(91.90)$ & $0.236(1.367 / 0.814-2.296)$ & $1542(89.96)$ & $204(86.44)$ & $0.097(0.711 / 0.474-1.066)$ \\
\hline $\mathrm{A}$ & $187(10.75)$ & $17(8.10)$ & & $172(10.04)$ & $32(13.56)$ & \\
\hline
\end{tabular}

${ }^{*} P<0.05$. SNP: single nucleotide polymorphism; AITD: autoimmune thyroid disease; OR: odds ratio; CI: confidence interval.

3.4. Genotyping-Phenotype Correlations. When next comparing the allele and genotype distributions of IL-22 polymorphisms the onset age between $\leq 18$ years old and $\geq 19$ years old, we found that genotype $\mathrm{A} / \mathrm{C}$ at $\mathrm{rs} 2046068, \mathrm{~T} / \mathrm{C}$ at rs2227478, and $\mathrm{C} / \mathrm{G}$ at rs1179251 were associated with the onset age in teenagers. Among them, A/C at rs2046068 and T/C at rs2227478 linked to the AITD adolescents ( $P=0.034$ and $P=0.028$, resp.). Besides, the frequency of allele $C$ at rs1179251 in AITD youngsters was more than that in AITD adults $(P=0.039, \mathrm{OR}=1.397)$. We further analyzed the allele and genotype frequencies of IL-22 SNPs with ophthalmopathy in AITD patients, and this study demonstrated that the SNP rs11611206 within the IL-22 gene was associated with thyroid-associated ophthalmopathy (TAO) $(P=0.023)$. Furthermore, the allele $\mathrm{G}$ in rs11611206 can decrease the risk of TAO by $28.9 \%(P=0.097)$. The above data were all shown in Table 5 .
As shown in Table 6, the similar stratification analysis method was conducted in the subgroup in GD and HT. Similarly, the genotypes CC in rs1179251 and GG in rs11611206 were correlated with Graves' ophthalmopathy (GO) $(P=0.036$ and $P=0.045$, resp.). In hypothyroidism patients with HT versus euthyroidism subjects with HT, the results showed the correlation with $G$ allele in rs11611206 $(P=0.024, \mathrm{OR}=1.828)$.

3.5. Haplotype Analysis. Figure 1 showed that haplotype analysis performed for the 5 SNPs revealed that only one block exists including rs2046068, rs2227478, rs2227485, and rs1179251. Table 7 displayed that there were three main haplotypes, namely, CATT, GACT, and CCCC, of these four loci. Through HaploView software examination, there was no association between those three haplotypes and AITD, $\mathrm{GD}$, and HT $(P>0.05)$. 
TABLE 6: Allele and genotype frequencies of IL-22 SNPs with ophthalmopathy in GD patients and hypothyroidism in HT patients.

\begin{tabular}{|c|c|c|c|c|c|c|}
\hline \multirow{2}{*}{ SNP loci } & \multicolumn{3}{|c|}{ Ophthalmopathy in GD } & \multicolumn{3}{|c|}{ Hypothyroidism in HT } \\
\hline & Without (\%) & With (\%) & $P$ value $(\mathrm{OR} / 95 \% \mathrm{CI})$ & Without (\%) & With (\%) & $P$ value $(\mathrm{OR} / 95 \% \mathrm{CI})$ \\
\hline \multicolumn{7}{|l|}{ rs2046068 } \\
\hline AA & $348(66.03)$ & $71(63.39)$ & 0.611 & $100(60.61)$ & $103(60.23)$ & 0.818 \\
\hline $\mathrm{CC}$ & $22(4.18)$ & $7(6.25)$ & & $9(5.45)$ & $7(4.09)$ & \\
\hline $\mathrm{AC}$ & $157(29.79)$ & $34(30.36)$ & & $56(33.94)$ & $61(35.68)$ & \\
\hline A & $853(80.93)$ & $176(78.57)$ & $0.418(0.864 / 0.606-1.231)$ & $256(77.58)$ & $267(78.07)$ & $0.877(1.029 / 0.715-1.481)$ \\
\hline $\mathrm{C}$ & $201(19.07)$ & $48(21.43)$ & & $74(22.42)$ & $75(21.93)$ & \\
\hline \multicolumn{7}{|l|}{ rs2227478 } \\
\hline $\mathrm{TT}$ & $347(65.84)$ & $71(63.39)$ & 0.558 & $101(61.21)$ & $103(60.23)$ & 0.789 \\
\hline $\mathrm{CC}$ & $21(3.98)$ & $7(6.25)$ & & $9(5.46)$ & $7(4.09)$ & \\
\hline TC & $159(30.18)$ & $34(30.36)$ & & $55(33.33)$ & $61(35.68)$ & \\
\hline $\mathrm{T}$ & $853(80.93)$ & $176(78.57)$ & $0.418(0.864 / 0.606-1.231)$ & $257(77.88)$ & $267(78.07)$ & $0.952(1.011 / 0.702-1.457)$ \\
\hline C & $201(19.07)$ & $48(21.43)$ & & $73(22.12)$ & $75(21.93)$ & \\
\hline \multicolumn{7}{|l|}{ rs2227485 } \\
\hline $\mathrm{CC}$ & $166(31.50)$ & $34(30.36)$ & 0.958 & 65 (39.39) & $50(29.24)$ & 0.139 \\
\hline $\mathrm{TT}$ & $124(23.53)$ & $26(23.21)$ & & $36(21.82)$ & $41(23.98)$ & \\
\hline $\mathrm{CT}$ & $237(44.97)$ & $52(46.43)$ & & $64(38.79)$ & $80(46.78)$ & \\
\hline $\mathrm{C}$ & $569(53.98)$ & $120(53.57)$ & $0.910(0.984 / 0.737-1.313)$ & $194(58.79)$ & $180(52.63)$ & $0.108(0.779 / 0.574-1.057)$ \\
\hline $\mathrm{T}$ & $485(46.02)$ & $104(46.43)$ & & $136(41.21)$ & $162(47.37)$ & \\
\hline \multicolumn{7}{|l|}{ rs1179251 } \\
\hline CC & $221(41.94)$ & $57(50.89)$ & $0.045^{*}$ & $69(41.82)$ & $80(46.78)$ & 0.173 \\
\hline GG & $60(11.38)$ & $17(15.18)$ & & $24(14.54)$ & $14(8.19)$ & \\
\hline CG & $246(46.68)$ & $38(33.93)$ & & $72(43.64)$ & $77(45.03)$ & \\
\hline $\mathrm{C}$ & $688(65.28)$ & $152(67.86)$ & $0.460(1.123 / 0.826-1.528)$ & $210(63.64)$ & $237(69.30)$ & $0.120(1.290 / 0.936-1.778)$ \\
\hline G & $366(34.72)$ & $72(32.14)$ & & $120(36.36)$ & $105(30.70)$ & \\
\hline \multicolumn{7}{|c|}{ rs11611206 } \\
\hline GG & $425(80.65)$ & $81(72.32)$ & $0.036^{*}$ & $128(77.57)$ & $148(86.55)$ & 0.086 \\
\hline $\mathrm{AA}$ & $7(1.33)$ & $0(0)$ & & $3(1.82)$ & $1(0.58)$ & \\
\hline GA & $95(18.02)$ & $31(27.68)$ & & $34(20.61)$ & $22(12.87)$ & \\
\hline G & $945(89.66)$ & $193(86.16)$ & $0.128(0.718 / 0.468-1.102)$ & $290(87.88)$ & $318(92.98)$ & $0.024^{*}(1.828 / 1.075-3.106)$ \\
\hline $\mathrm{A}$ & $109(10.34)$ & $31(13.84)$ & & $40(12.12)$ & $24(7.02)$ & \\
\hline
\end{tabular}

${ }^{*} P<0.05$. SNP: single nucleotide polymorphism; GD: Graves' disease; HT: Hashimoto's thyroiditis; OR: odds ratio; CI: confidence interval.

\section{Discussion}

Recently, it is clear that AITD has become one of the most important autoimmune healthcare in the population. However, the pathogenesis of AITD remains elusive. As we all know, the occurrence of AITD is with obvious sex tendency, and this disease is more popular in women with the ratio of female to male being $5: 1$ to $10: 1$ [13]. The disease also displays a family aggregation phenomenon, as there are $40 \%-50 \%$ of patients with their families suffering from thyroid disease [14]. Increasing data have demonstrated that environment factors, immune elements, and genetic susceptibility are all involved in the etiology of this disease $[15,16]$.

IL-22, as a member of IL-10 cytokine superfamily, is mainly secreted by Th22 cells and also by their dominant functional cytokine [17]. Our precious publication about a functional study to explore the possible biological mechanisms underlying the association between IL-22 and AITD has indicated that serum IL-22, the mRNA expression of IL-22, and Th22 cells are all increased in GD patients, implying that IL-22 as a proinflammatory factor participates in onset and development of AITD [9]. Recent studies have demonstrated associations between several variants of IL-22 and multiple immune-related diseases $[7,8,11,18]$. In the present study, we attempted to find out the potential link of polymorphisms of IL-22 with the risk of AITD.

There were no significant differences among the five IL-22 gene polymorphism distribution in the whole AITD, GD, and HT subjects and controls, except that there was a weak association between allele A of rs2046068 and HT patients (although with no statistical significance). Because of the gender trend in AITD [13], we subsequently conducted the sex-stratified analysis and indeed found some interesting and significant results through gender-stratified comparison. The results of our case-control study discovered that several loci of IL-22 are associated with the susceptibility of AITD 

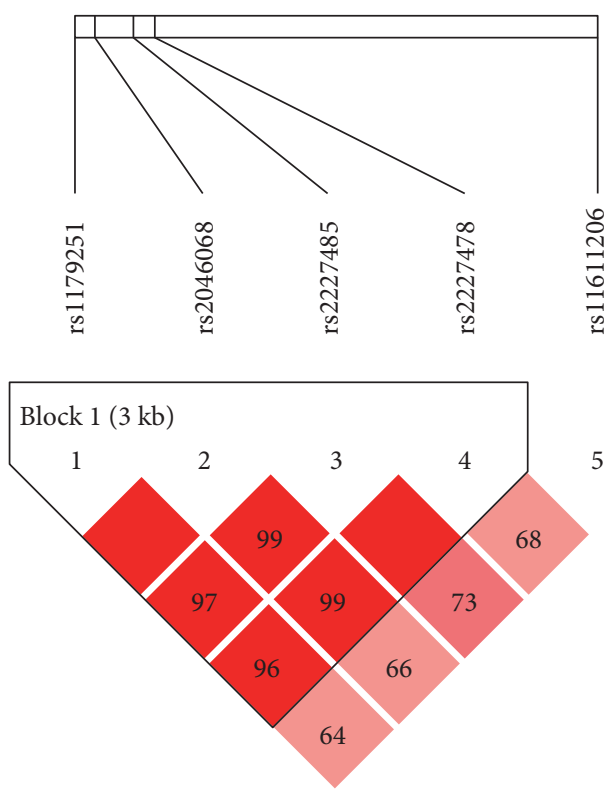

Figure 1: Haplotype by HaploView software analyses.

TABLE 7: Distribution of haplotypes for IL-22 gene polymorphisms in AITD, GD, and HT patients and controls groups.

\begin{tabular}{lcccccccc}
\hline & & & & \multicolumn{4}{c}{$P$ value for association } \\
Haplotypes & AITD & GD & HT & NC & AITD & GD & HT \\
& & & & & versus & versus & versus \\
& & & & & & NC & NC \\
\hline CATT & 887 & 589 & 298 & 794 & 0.429 & 0.693 & 0.294 \\
GACT & 662 & 438 & 224 & 573 & 0.914 & 0.807 & 0.841 \\
CCCC & 395 & 247 & 148 & 321 & 0.310 & 0.783 & 0.087 \\
\hline
\end{tabular}

AITD: autoimmune thyroid disease; GD: Graves' disease; HT: Hashimoto's thyroiditis; NC: normal controls.

in the Han Chinese population. For instance, genotype GG in rs1179251 linked to the female AITD and allele A in rs2046068 and allele $\mathrm{T}$ in rs2227478 decreased the risk to HT by $44.3 \%$ and $43.6 \%$, respectively, and allele C in rs 1179251 increased the predisposition for HT by $73.9 \%$ in male patients. The current study and our precious report [9] both imply that IL-22 participates in the pathogenesis of AITD, through polymorphisms and aberrant expression. There is no doubt that IL-22 as a vital cytokine takes part in the immune dysfunction of AITD, and as a key gene member, it will be added to the list of genes which influences the predisposition for AITD.

Subsequent phenotype analyses further confirmed the association between IL-22 variants and AITD subphenotypes. In teenagers, the onset of AITD is often with much obvious family history [14], which was also identified in our study that showed that the percentage of family history in teenager patients $(30.48 \%)$ was higher than that in adult patients $(12.41 \%)$. Our current research showed that genotype AA in rs2046068 and genotype TT in rs2227478 were correlated with the risk towards teenagers to develop AITD. Besides, it showed that allele $\mathrm{C}$ of rs1179251 increases the teenagers' risk for AITD by 39.7\%. Similarly, a pervious paper of ours also reported that there is an association between TNFSF4 gene variations and AITD adolescents [19]. So these results strongly suggest that the occurrence of thyroid dysfunction in teenagers can be caused by family history, which means the genetic background. The eye disorder associated with AITD, called thyroid-associated ophthalmopathy, drastically reduces the quality of life in affected patients and even contributes to the disability of patients [20]. Enormous amounts of documents have shown that TAO is a complex disease with multifaceted mechanism including smoking history, thyroid dysfunction, and positive TRAb $[21,22]$. We showed for the first time that genotype GG in rs11611206 within IL-22 gene is associated with TAO. When it comes to TAO, most people think first of Graves' orbitopathy (GO), as more than $80 \%$ TAO patients also present with Graves' disease [23]. In our present study, as for GO, the SNPs in IL-22 at both rs1179251 (genotype CC) and rs11611206 (genotype GG) linked to GO. Autoimmune hypothyroidism is largely caused by Hashimoto's thyroiditis, in which irregulatory immune responses against to the thyroid tissue happens [24]; therefore, evaluation of thyroid function in HT is of particular importance. Our results suggested that rs11611206 polymorphism of IL-22, including both allele $\mathrm{G}$ and genotype GG, may be a genetic risk factor for autoimmune hypothyroidism in HT. Thus polymorphisms in the IL-22 gene confer predisposition to some subclasses of AITD.

This is the first report about the IL-22 gene polymorphisms in AITD. Also, this is the first study on the link between the loci in the IL-22 region and AITD susceptibility in Chinese population. Further case-control researches with larger populations and more races are needed to identify these observations.

\section{Conclusion}

Current findings indicated that polymorphisms in IL-22 may explain part of the AITD genetic tendency. To be sure, IL-22 is a risk factor for the development of AITD.

\section{Ethical Approval}

This work was performed under the approval of the Ethics Committee of Jinshan Hospital of Fudan University.

\section{Conflicts of Interest}

The authors declare that they have no conflict of interest and are responsible for the content of this paper.

\section{Authors' Contributions}

Jin-an Zhang conceived and designed the experiments. Rong-hua Song conducted this study and analyzed the data. Wen Wang, Qian Li, and Qiu-ming Yao collected the specimens. Xiao-qing Shao is responsible for the extraction of DNA samples. Rong-hua Song wrote the main 
manuscript text. Jin-an Zhang helped revise the manuscript. All authors reviewed the manuscript.

\section{Acknowledgments}

The authors would like to acknowledge each patient and control subject who participated in this study. This present project is supported by grants from the National Natural Science Foundation of China (Grant nos. 81471004 and 81670722) and the Key Disciplines Development of Shanghai Jinshan District (no. JSZK2015A02).

\section{References}

[1] M. J. Simmonds, "GWAS in autoimmune thyroid disease: redefining our understanding of pathogenesis," Nature Reviews Endocrinology, vol. 9, no. 5, pp. 277-287, 2013.

[2] W. S. Yoo and H. K. Chung, "Recent advances in autoimmune thyroid diseases," Endocrinology and Metabolism, vol. 31, no. 3, pp. 379-385, 2016.

[3] R. H. Song, Z. Y. Yu, Q. Wang et al., "Polymorphisms of the TNFAIP3 region and Graves' disease," Autoimmunity, vol. 47, no. 7, pp. 459-465, 2014.

[4] N. Yan, S. Meng, R. H. Song et al., "Polymorphism of IL37 gene as a protective factor for autoimmune thyroid disease," Journal of Molecular Endocrinology, vol. 55, no. 3, pp. 209218, 2015.

[5] R. H. Song, Q. Qin, N. Yan et al., "Variants in IRAK1MECP2 region confer susceptibility to autoimmune thyroid diseases," Molecular and Cellular Endocrinology, vol. 399, pp. 244-249, 2015.

[6] M. Perusina Lanfranca, Y. Lin, J. Fang, W. Zou, and T. Frankel, "Biological and pathological activities of interleukin-22," Journal of Molecular Medicine, vol. 94, no. 5, pp. 523-534, 2016.

[7] H. Saeki, T. Hirota, H. Nakagawa et al., "Genetic polymorphisms in the IL22 gene are associated with psoriasis vulgaris in a Japanese population," Journal of Dermatological Science, vol. 71, no. 2, pp. 148-150, 2013.

[8] L. M. Medrano, M. Garcia-Magarinos, B. Dema et al., "Th17related genes and celiac disease susceptibility," PLoS One, vol. 7, no. 2, article e31244, 2012.

[9] R. H. Song, Z. Y. Yu, Q. Qin et al., "Different levels of circulating Th22 cell and its related molecules in Graves' disease and Hashimoto's thyroiditis," International Journal of Clinical and Experimental Pathology, vol. 7, no. 7, pp. 4024-4031, 2014.

[10] R. H. Song, Q. Qin, X. Wang et al., "Differential cytokine expression detected by protein microarray screening in peripheral blood of patients with refractory Graves' disease," Clinical Endocrinology, vol. 84, no. 3, pp. 402-407, 2016.

[11] G. Zhang, X. Chen, L. Chan et al., “An SNP selection strategy identified IL-22 associating with susceptibility to tuberculosis in Chinese," Scientific Reports, vol. 1, p. 20, 2011.

[12] L. Bartalena, L. Baldeschi, A. J. Dickinson et al., "Consensus statement of the European group on Graves' orbitopathy (EUGOGO) on management of Graves' orbitopathy," Thyroid, vol. 18, no. 3, pp. 333-346, 2008.

[13] G. Effraimidis and W. M. Wiersinga, "Mechanisms in endocrinology: autoimmune thyroid disease: old and new players," European Journal of Endocrinology, vol. 170, no. 6, pp. R241-R252, 2014.
[14] N. Manji, J. D. Carr-Smith, K. Boelaert et al., "Influences of age, gender, smoking, and family history on autoimmune thyroid disease phenotype," The Journal of Clinical Endocrinology and Metabolism, vol. 91, no. 12, pp. 4873-4880, 2006.

[15] W. M. Wiersinga, "Clinical relevance of environmental factors in the pathogenesis of autoimmune thyroid disease," Endocrinology and Metabolism, vol. 31, no. 2, pp. 213-222, 2016.

[16] B. Kristensen, "Regulatory B and T cell responses in patients with autoimmune thyroid disease and healthy controls," Danish Medical Journal, vol. 63, no. 2, 2016.

[17] T. Duhen, R. Geiger, D. Jarrossay, A. Lanzavecchia, and F. Sallusto, "Production of interleukin 22 but not interleukin 17 by a subset of human skin-homing memory T cells," Nature Immunology, vol. 10, no. 8, pp. 857-863, 2009.

[18] G. G. Ding, G. L. Zhang, X. C. Chen, M. X. Zhang, L. Yang, and Z. Wang, "A study on the relationship between single nucleotide polymorphisms of interleukin-22 and susceptibility to pulmonary tuberculosis," Zhonghua Jie He He Hu xi $\mathrm{Za}$ Zhi = Zhonghua Jiehe He Hu Xi Za Zhi = Chinese Journal of Tuberculosis and Respiratory Diseases, vol. 35, no. 8, pp. 596-600, 2012.

[19] R. H. Song, Q. Wang, Q. M. Yao et al., “TNFSF4 gene variations are related to early-onset autoimmune thyroid diseases and hypothyroidism of Hashimoto's thyroiditis," International Journal of Molecular Sciences, vol. 17, no. 8, 2016.

[20] H. Lahooti, D. Cultrone, S. Edirimanne et al., "Novel singlenucleotide polymorphisms in the calsequestrin-1 gene are associated with Graves' ophthalmopathy and Hashimoto's thyroiditis," Clinical Ophthalmology, vol. 9, pp. 1731-1740, 2015.

[21] D. L. Weiler, "Thyroid eye disease: a review," Clinical \& Experimental Optometry, vol. 100, no. 1, pp. 20-25, 2017.

[22] D. Huang, N. Xu, Y. Song, P. Wang, and H. Yang, "Inflammatory cytokine profiles in the tears of thyroid-associated ophthalmopathy," Graefe's Archive for Clinical and Experimental Ophthalmology = Albrecht von Graefes Archiv fur Klinische und Experimentelle Ophthalmologie, vol. 250, no. 4, pp. 619-625, 2012.

[23] J. Shen, Z. Li, W. Li et al., "Th1, Th2, and Th17 cytokine involvement in thyroid associated ophthalmopathy," Disease Markers, vol. 2015, Article ID 609593, 6 pages, 2015.

[24] H. Patel, M. S. Mansuri, M. Singh, R. Begum, M. Shastri, and A. Misra, "Association of cytotoxic T-lymphocyte antigen 4 (CTLA4) and thyroglobulin (TG) genetic variants with autoimmune hypothyroidism," PLoS One, vol. 11, no. 3, article e0149441, 2016. 


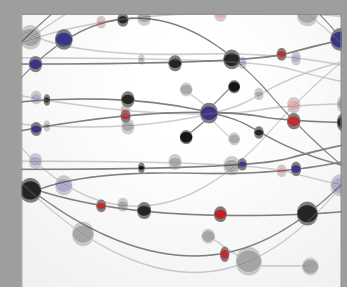

The Scientific World Journal
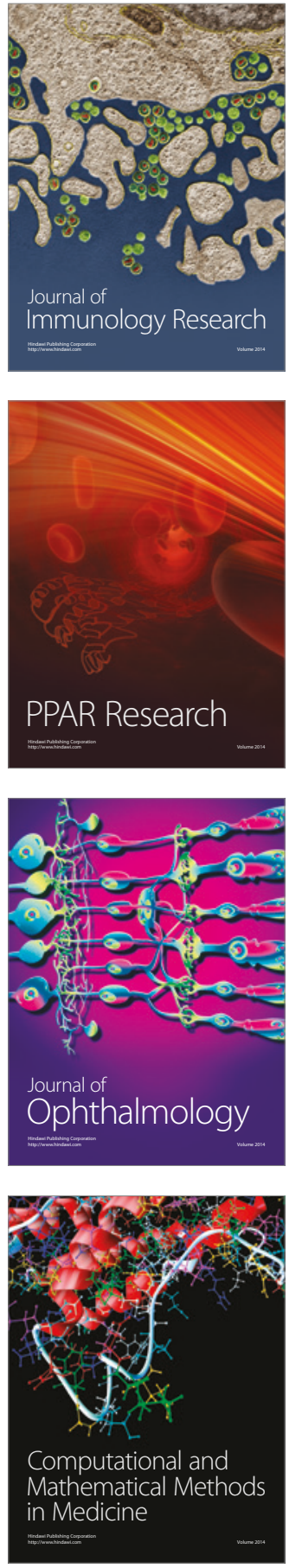

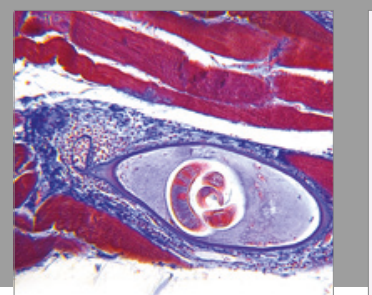

Gastroenterology Research and Practice
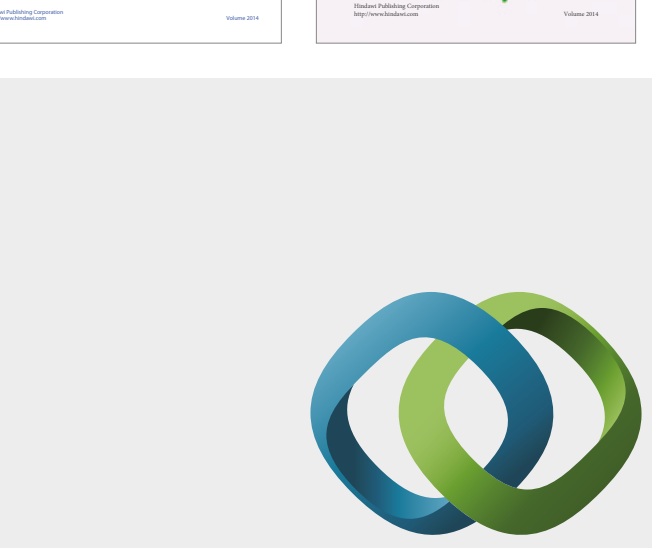

\section{Hindawi}

Submit your manuscripts at

https://www.hindawi.com
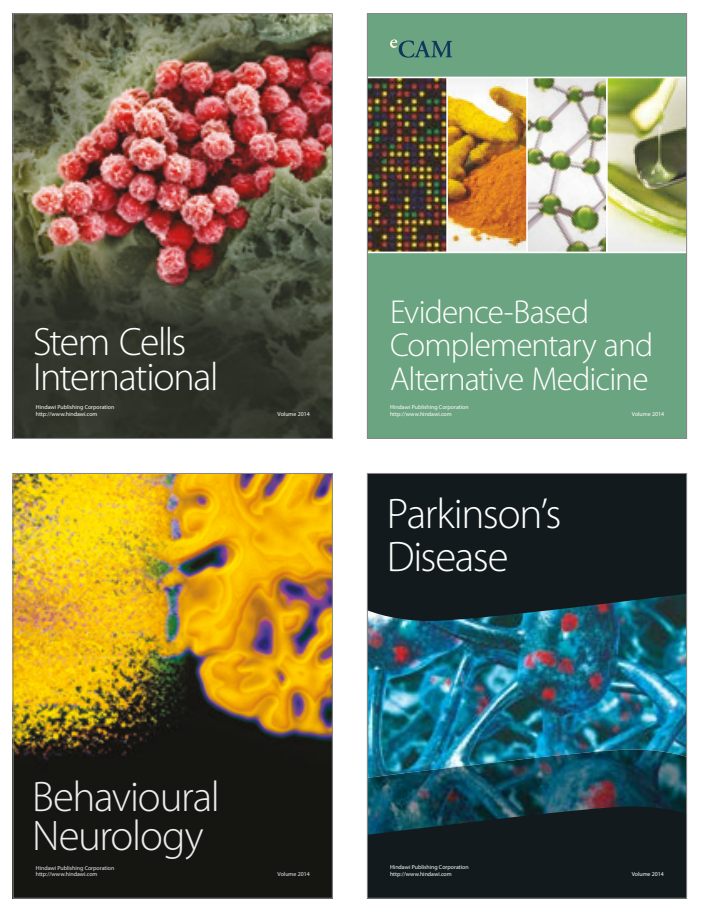
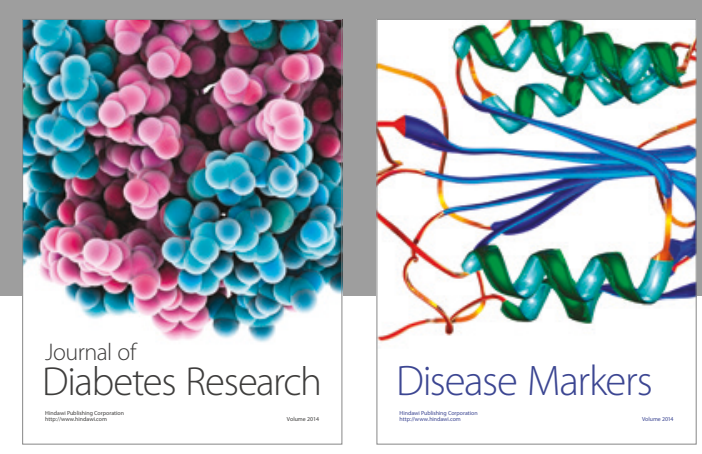

Disease Markers
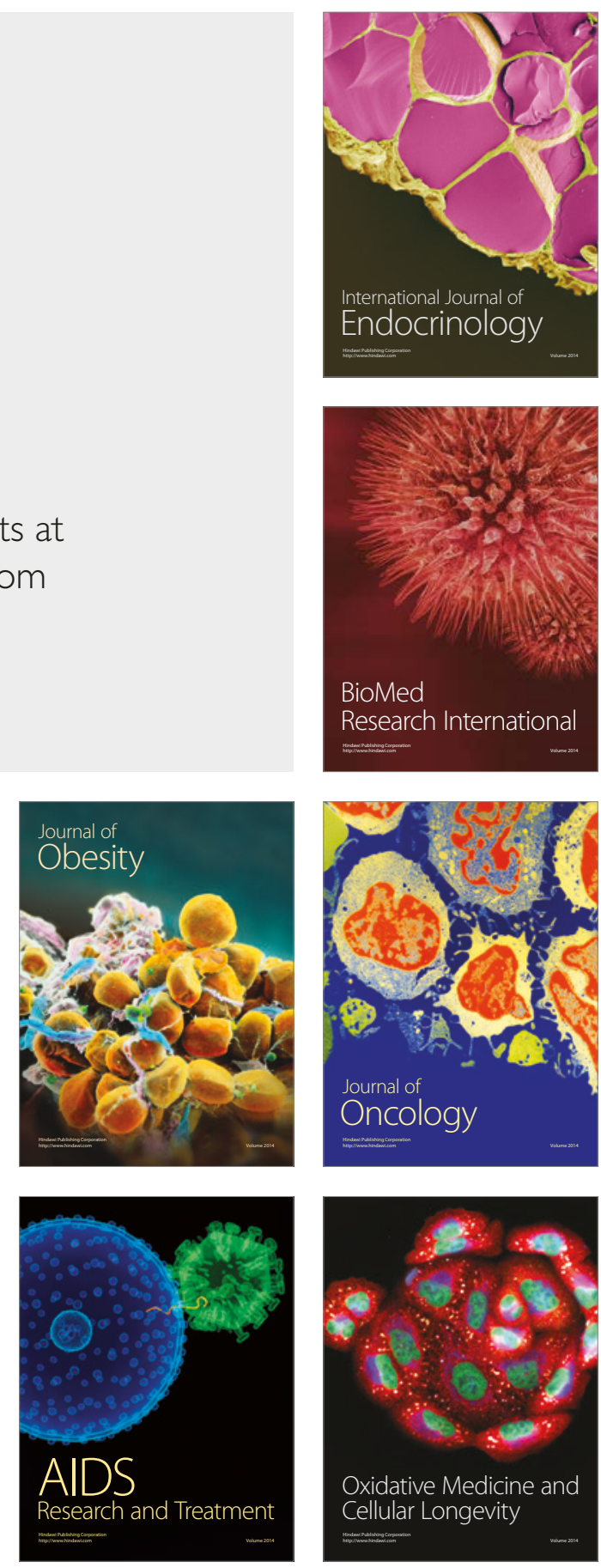